\title{
BMJ Open Screening strategies for hypertension: a systematic review protocol
}

To cite: Schmidt B-M, Durão S, Toews I, et al. Screening strategies for hypertension: a systematic review protocol. BMJ Open 2019;9:e025043. doi:10.1136/ bmjopen-2018-025043

- Prepublication history and additional material for this paper are available online. To view these files, please visit the journal online (http://dx.doi. org/10.1136/bmjopen-2018025043).

Received 27 June 2018 Revised 29 0ctober 2018 Accepted 30 October 2018

Check for updates

(C) Author(s) (or their employer(s)) 2019. Re-use permitted under CC BY-NC. No commercial re-use. See rights and permissions. Published by BMJ.

${ }^{1}$ Cochrane South Africa, South African Medical Research

Council, Cape Town, South Africa ${ }^{2}$ Institute for Evidence in Medicine (for Cochrane Germany Foundation), Medical Center-University of Freiburg, Freiburg, Germany

${ }^{3}$ College of Medicine and Health Science, University of Rwanda, Kigali, Rwanda

${ }^{4}$ Division of Clinical

Pharmacology, Department of Medicine, Faculty of Medicine and Health Sciences, University of Stellenbosch, Cape Town, South Africa

Correspondence to

Dr Tamara Kredo;

tamara.kredo@mrc.ac.za

\author{
Bey-Marrié Schmidt, ${ }^{1}$ Solange Durão, ${ }^{1}$ Ingrid Toews, ${ }^{2}$ Charlotte M Bavuma, ${ }^{3}$ \\ Joerg J Meerpohl, ${ }^{2}$ Tamara Kredo ${ }^{1,4}$
}

\begin{abstract}
Introduction It is unclear whether early detection of hypertension, through screening, leads to healthier behaviours and better control of blood pressure levels. There is a need to learn from studies that have assessed the impact of different screening approaches on patient important outcomes. This systematic review protocol outlines the methods that will be used to assess the comparative effectiveness of different screening strategies (mass, targeted or opportunistic) for hypertension to reduce morbidity and mortality associated with hypertension.
\end{abstract}

Methods and analysis We will primarily search Cochrane Central Register of Controlled Trials, Medline, Embase and Latin American and Caribbean Health Sciences Literature (LILACS). Relevant randomised controlled trials, controlled before and after, interrupted time series and prospective analytic cohort studies regardless of publication date, language and geographic location, will be included. We are interested in clinical, adverse event and health system outcomes. Two reviewers will independently screen titles, abstracts and full-text articles against inclusion criteria; perform data extraction and assess risk of bias in included studies. We will assess the certainty of the overall evidence using the Grading of Recommendations Assessment, Development and Evaluation approach and report findings accordingly.

Ethics and dissemination No ethics approval will be sought, as only secondary studies will be used. Findings will be disseminated through peer-reviewed publication and conference presentations.

PROSPERO registration number CRD42018093046.

\section{BACKGROUND}

\section{Description of the condition}

Hypertension, also known as raised or high blood pressure, is a long-term non-communicable medical condition, where the blood pressure in the arteries is persistently elevated. ${ }^{1}$ Blood pressure can be expressed as two measurements, systolic blood (SBP) and diastolic blood (DBP) pressures which are the minimum and maximum pressures. Table 1 compares previous versus new thresholds for high blood pressures. ${ }^{2}$

Over the long term, hypertension is a major risk factor for cardiovascular events, such as heart disease, stroke and kidney failure, and
Strengths and limitations of this study

- This systematic review is a response to priority setting conducted in collaboration with policy-makers who recognised a gap in available synthesised evidence regarding approaches for hypertension screening (mass, opportunities or targeted screening strategies).

- This review will include randomised and non-randomised controlled studies to capture all relevant evidence regarding programmes of hypertension screening.

- We will conduct a comprehensive search across several databases without restricting for language or publication status.

- We plan to meta-analyse outcome data; however, included studies may vary in terms of study design and the outcomes reported, and therefore we may present narrative evidence syntheses.

The review authors have complementary expertise in systematic review methods and content which will ensure a review that is relevant for policy and practice.

disability and premature mortality. ${ }^{3}$ Factors that increase the risk of high blood pressure include genetic and lifestyle factors such as excessive salt and fat consumption, physical inactivity, harmful alcohol consumption and poor management of stress. ${ }^{3}$ There is growing evidence that younger people (such as adolescents) are also at risk of hypertension because of these lifestyle factors. ${ }^{45}$

Hypertension is a major public health problem; it is the most common cardiovascular disorder affecting approximately one billion people globally, and remains (since the early 2000s) the single leading contributor to the global burden of morbidity and mortality. ${ }^{1}$ In Sub-Saharan Africa, it is estimated that 10-20million people out of approximately 650 million people may have hypertension. ${ }^{6}$ This high prevalence of hypertension is attributed to population growth (migration from rural to urban areas), changes in dietary habits, ageing of the population and social stress. ${ }^{13}$ A large proportion 


\begin{tabular}{|c|c|c|}
\hline BP category & SBP & DBP \\
\hline \multicolumn{3}{|l|}{ Previous guidelines } \\
\hline High & $\geq 140 \mathrm{~mm} \mathrm{Hg}$ and & $\geq 90 \mathrm{~mm} \mathrm{Hg}$ \\
\hline \multicolumn{3}{|l|}{ New guidelines } \\
\hline Normal & $<120 \mathrm{~mm} \mathrm{Hg}$ and & $<80 \mathrm{~mm} \mathrm{Hg}$ \\
\hline Elevated & $120-129 \mathrm{~mm} \mathrm{Hg}$ and & $<80 \mathrm{~mm} \mathrm{Hg}$ \\
\hline Hypertension & $\begin{array}{l}\text { Stage 1: } 130- \\
139 \mathrm{~mm} \mathrm{Hg} \text { or } \\
\text { Stage 2: } \geq 140 \mathrm{~mm} \\
\text { Hg or }\end{array}$ & $\begin{array}{l}80-89 \mathrm{~mm} \mathrm{Hg} \\
\geq 90 \mathrm{~mm} \mathrm{Hg}\end{array}$ \\
\hline Hypertensive crisis & $>180 \mathrm{~mm} \mathrm{Hg}$ and/or & $>120 \mathrm{~mm} \mathrm{Hg}$ \\
\hline
\end{tabular}

BP, blood pressure; DBP, diastolic blood pressure; SBP, systolic blood pressure.

of the population with hypertension remains undiagnosed, untreated or inadequately treated, contributing to the rising burden of cardiovascular disease. ${ }^{7}$

\section{Description of the intervention}

Screening programmes for hypertension could help reduce morbidity and mortality linked to it. ${ }^{38}$ Screening is generally defined as the detection of unknown disease among apparently healthy individuals by means of tests or examinations conducted to identify those at an increased risk for the condition. ${ }^{9}$
Various devices (electronic, mercury and aneroid) can be used to measure blood pressure, with semiautomatic devices being the most reliable, because readings can be taken even when batteries run low, which may be a common problem in resource-limited settings. ${ }^{3}$ Two blood pressure measurements should be recorded daily for several days. These measurements should be taken at least a minute apart, ideally in the morning and again in the evening while the person is seated. For accuracy, measurements taken on the first day are discarded, and an average is taken of all the remaining measurements to confirm diagnosis of hypertension. ${ }^{3}$ It is common practice that the diagnosis of hypertension is confirmed if the resting blood pressure is persistently at $\mathrm{SBP} \geq 130 / 140$ or $\mathrm{DBP} \geq 80 / 90 \mathrm{~mm} \mathrm{Hg} .{ }^{3} 10$ This review will primarily focus on screening strategies for hypertension and not be on the thresholds used for diagnosis; however, we will note blood pressure thresholds as defined by the authors of included studies.

Key components of screening programmes for hypertension include equipment and trained health professionals and patient education and informed consent, and good relationships between health professions (which are beneficial for referral processes between different healthcare facilities or services). ${ }^{3}$ These components make screening for hypertension (across an entire population) a costly intervention, because of the lengthy time to diagnosis and the human and financial resources required.

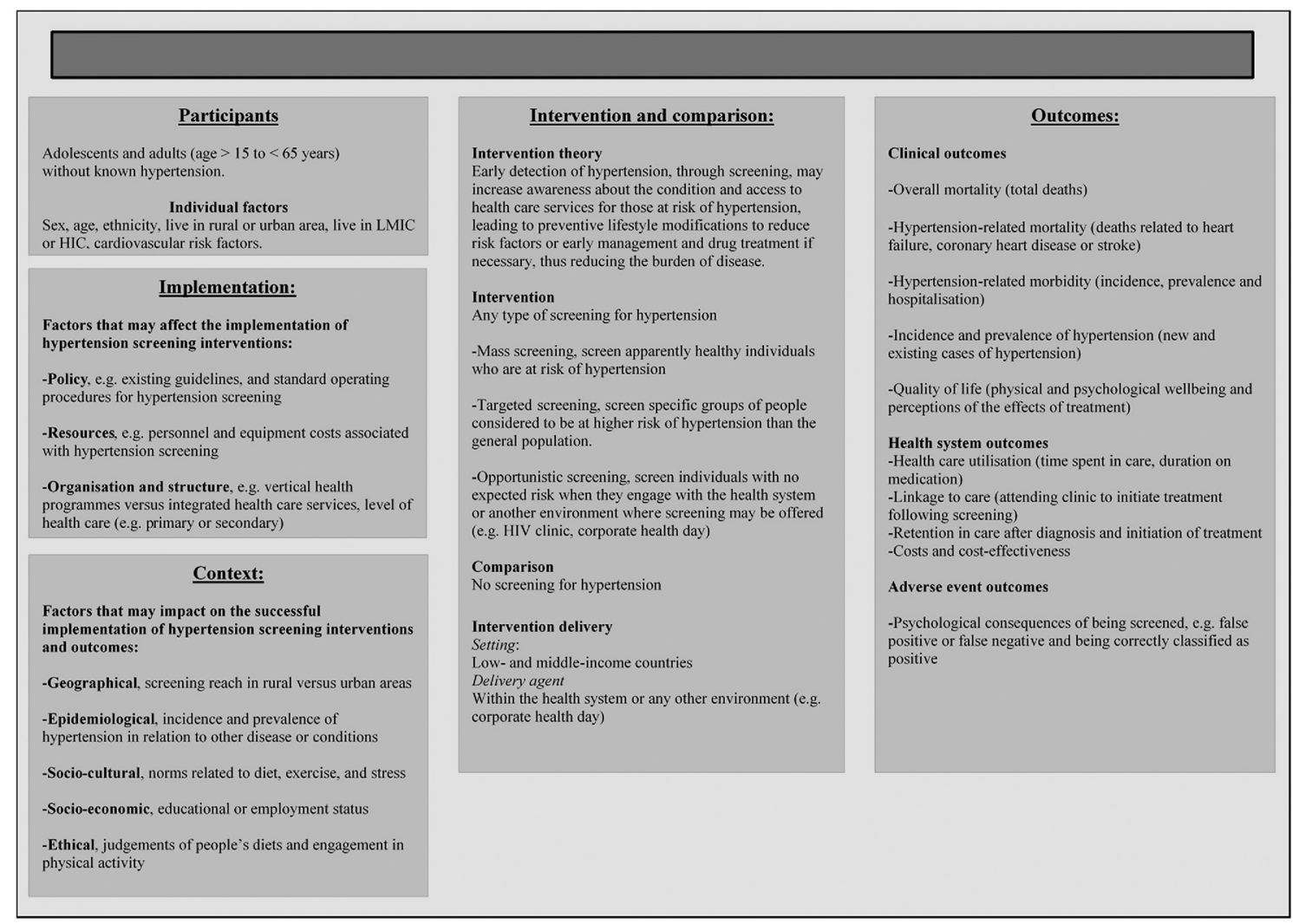

Figure 1 Screening for hypertension. HIC, high-income countries. 


\section{How the intervention might work}

The logic model ${ }^{11}$ in figure 1 outlines how hypertension screening may reduce the burden of disease considering participant, intervention, implementation and contextual factors. Early detection of hypertension through screening could increase awareness for those at risk of hypertension, and thus lead to preventative action or early management, which may ultimately curb the societal and economic burden of the disease. ${ }^{7}$

\section{Why is it important to do this review?}

Interventions to prevent or manage hypertension should be feasible, affordable, sustainable and effective. Thus, vertical programmes that focus solely on hypertension are not consistently recommended. ${ }^{3}$ Early detection of hypertension may be a critical element for containing health-related costs, especially when screening for hypertension is offered as a point-of-care or integrated service. However, hypertension is primarily associated with behavioural and socioeconomic risk factors; therefore, early detection of mild hypertension may not significantly impact health-related costs in the long term or improve health outcomes. Additionally, the factors associated with hypertension are generally the problems of urban areas in resource-limited countries; therefore, preventing hypertension may involve other stakeholders (eg, policy-makers) beyond screening by health professionals. ${ }^{3} 12$

Since it is unclear whether screening for hypertension leads to healthier behaviours and better control of blood pressure levels, it is important to learn from studies that have assessed the impact of screening on hypertension outcomes. A 2014 systematic review supported the U.S. Preventive Services Task Force in updating its recommendation on screening for high blood pressure in adults. This review focused on the role of confirming hypertension diagnoses, rescreening intervals and ambulatory blood pressure monitoring and home blood pressure monitoring. The evidence from this systematic review does not provide guidance on different screening strategies.

A recent overview of systematic reviews, on diabetes and hypertension screening programmes, found that there is a need for a systematic review to assess the effectiveness and impact of various screening interventions. ${ }^{13}$ This review aims to address this gap in the literature, with a specific focus on evidence from resource-limited countries, where the behavioural and socioeconomic risk factors of hypertension are similar to the broader problems of urban areas in these countries. This will provide clarity on whether screening of hypertension, in all age groups, will contain health-related costs and improve outcomes related to hypertension and associated life-threatening complications.

\section{Objectives}

To assess the comparative effectiveness of different screening strategies, mass, targeted or opportunistic, for hypertension to reduce morbidity and mortality associated with hypertension.

\section{METHODS}

\section{Criteria for considering studies for this review}

Types of studies

We will include randomised controlled trials (RCTs) and non-randomised controlled trials (NRCTs), ${ }^{14}$ that is, controlled before and after (CBA), interrupted time series (ITS) and prospective analytic cohort studies. We do not expect to find many RCTs, so NRCTs will also be included in the review, given the programmatic nature of screening for hypertension. RCTs are experimental studies in which people are randomly allocated to one of two or more groups receiving an intervention or control treatment or no treatment. CBA is a type of non-randomised study in which outcomes are measured before and after a treatment, both in a group that receives the treatment and in another comparison group. ITS is also a type of non-randomised study that measures an outcome at multiple time points before and after an intervention (the 'interruption'). The design attempts to detect whether the intervention has had an effect greater than any underlying trend over time. ITS studies should be controlled and they must have at least three data points before and after a clearly defined intervention in terms of content and timing. ${ }^{15}$ The last type of non-randomised study that we will include is prospective analytic cohort studies, where participants are already either exposed or unexposed to an intervention, but without the outcome of interest at the start of the study, because participants are followed forward in time after which outcomes are measured. There should be at least two arms for the cohort to provide a comparison of the exposure of interest.

We will include studies regardless of their language or publication status.

\section{Types of participants and setting}

Participants are healthy adolescents, adults and elderly persons (aged between 15 and 65+) without known hypertension. We will include studies where participants present with risk factors for hypertension.

\section{Types of interventions and comparator}

Studies on mass, targeted or opportunistic hypertension screening will be eligible. The interventions must be compared with no screening and participants must be followed for at least 1 year.

Mass screening involves screening apparently healthy populations regardless of risk factors (at public places, for example, markets); targeted screening involves screening specific groups of people who are considered to be at higher risk of hypertension than the general population; and opportunistic screening involves screening individuals engaging with the health system or another environment where screening may be offered (eg, HIV clinic, corporate health day).

Types of outcome measures

Clinical

1. Overall mortality (total deaths). 
2. Hypertension-related mortality (deaths related to heart failure, coronary heart disease, stroke or endstage kidney disease).

3. Hypertension-related morbidity (incidence, prevalence and hospitalisation due to stroke, coronary heart disease or end-stage renal disease).

4. Incidence and prevalence of hypertension (ratio of detected hypertension to expected prevalence of hypertension).

5. Quality of life (physical and psychological well-being and perceptions of the effects of treatment).

Health system

6. Healthcare utilisation (time spent in care, duration on medication).

7. Linkage to care (attending clinic to initiate treatment following screening).

8. Retention in care after diagnosis and initiation of treatment.

9. Costs and cost effectiveness.

Adverse events of being screened

10. Psychological consequences of being screened, for example, false positive or false negative and being correctly classified as positive (new diagnosis)

\section{Search methods for identification of studies}

\section{Electronic searches}

The Cochrane Hypertension information specialist will search the following databases without language, publication year or publication status restrictions:

- The Cochrane Hypertension Specialised Register via the Cochrane Register of Studies (CRS-Web).

- The Cochrane Central Register of Controlled Trials via the CRS-Web.

- Medline Ovid (from 1946 onwards), Medline Ovid Epub Ahead of Print, and Medline Ovid In-Process and Other Non-Indexed Citations.

- Embase Ovid (from 1974 onwards).

- LILACS Bireme (from 1982 onwards).

- ClinicalTrials.gov (www.clinicaltrials.gov).

- WHO International Clinical Trials Registry Platform ( www.who.it.trialsearch).

The subject strategies for databases will be modelled on the search strategy designed for Medline in online appendix 1. Where appropriate, these will be combined with subject strategy adaptations of the sensitivity-maximising and precision-maximising search strategy designed by Cochrane for identifying RCTs (as described in the Cochrane Handbook for Systematic Reviews of Interventions V.5.1.0, Box 6.4.c. (Handbook 2011)). Search terms for non-randomised trials are based on the Effective Practice and Organisation of Care (EPOC) search filter for Ovid Medline. Full strategies for the above databases will be published in the review.

\section{Searching other resources}

- The information specialist will search the Cochrane Hypertension Specialised Register segment (which includes searches of Medline, Embase, The Cochrane
Library and Epistemonikos for systematic reviews) to retrieve published systematic reviews related to this review title, so that we can scan their reference lists to identify additional relevant trials. The Specialised Register also includes searches of the Allied and Complementary Medicine Database, CAB Abstracts and Global Health, Cumulative Index to Nursing and Applied Health Literature (CINAHL), ProQuest Dissertations and Theses and Web of Science.

- We will check the bibliographies of included studies and any relevant systematic reviews identified for further references to relevant trials.

- We will contact experts/organisations in the field to obtain additional information on relevant trials.

- We may contact original authors for clarification and further data if trial reports are unclear.

\section{Data collection and analysis}

Selection of studies

At least, two review authors will independently screen all titles or abstracts, or both, of all records retrieved to determine their eligibility for full-text screening. Full texts of potentially eligible or unclear studies will be retrieved and assessed for inclusion into the review by two researchers independently. Any disagreements will be resolved through rechecking the full text and/ or discussion with a third reviewer. We will present a Preferred Reporting Items for Systematic Reviews and Meta-Analyses flow diagram and a table of excluded studies and explanations for exclusion, in the review. ${ }^{16}$

\section{Data extraction and management}

We will pilot the data extraction form on two included studies to ensure information is captured in a standard manner. Two review authors will independently extract study data related to participants, intervention, comparison and outcome characteristics using the standard data extraction form. We will record any missing information in order to contact the author of the primary study. Any disagreements will be resolved through discussion or in consultation with a third reviewer.

\section{Assessment of risk of bias in included studies}

The risk of bias assessment tool modified by the Cochrane Effective Practice and Organisation of Care (EPOC) Group will be used. ${ }^{13}$ It is widely used and validated for systematic reviews including a wide range of study designs. Review authors will independently assess the risk of bias in included studies. Any disagreements will be resolved through discussion or in consultation with a third reviewer. We will judge individual studies to have 'low', 'unclear' or 'high' risk of bias. Low risk of bias is plausible bias unlikely to alter results, unclear risk of bias is plausible bias that raises some doubt about the results and high risk of bias is plausible bias that seriously weakens confidence in results. We will follow the recommendation by EPOC to score NRCTs as 'high' risk of bias. ${ }^{17}$ 
The following criteria will be applied to assess the risk of bias assessment in RCTs and NRCTs:

- Was the allocation sequence adequately generated? (RCTs)

- Was the allocation adequately concealed? (RCTs)

- Were baseline outcome measurements similar? (All)

- Were baseline characteristics similar? (All)

- Were incomplete outcome data adequately addressed? (RCTs)

- Was the knowledge of the allocated intervention adequately prevented during the study? (RCTs)

- Was the study adequately protected against contamination? (RCTs)

- Was the study free from selective outcome reporting? (RCTs)

- Was the study free from other risks of bias? (All)

For ITS, the risk of bias assessment will be based on the following criteria:

- Was the intervention independent of other changes?

- Was the shape of the intervention effect pre-specified?

-Was the intervention unlikely to affect data collection?

- Was knowledge of the allocated interventions adequately prevented during the study?

- Were incomplete outcome data adequately addressed?

- Was the study free from selective outcome reporting?

- Was the study free from other risks of bias?

\section{Measures of treatment effect}

We will present dichotomous outcomes as risk ratios, and continuous outcomes as mean differences (MD) with SD between the change in the intervention and control groups if the outcomes have been measured in the same way across all studies. In the case that continuous outcomes have been measured in different ways across studies, then we will use the standardised MD between the intervention and control groups. We will present time-to-event outcomes as HR. We will report 95\% CIs for all outcomes.

\section{Unit of analysis issues}

We will consider the level at which randomisation occurred (eg, in cluster-randomised trials, groups of individuals may be randomised together to the same intervention), and where repeated measurements are taken, there may be multiple observations for the same outcome. ${ }^{18}$ In the case that more than one comparison is available from the same study, we will combine groups into a single pair comparison. If included cluster-randomised trials have not appropriately adjusted for the clustering of participants in their analysis, then we will attempt to reanalyse them. The design effect (of cluster-randomised trials) may lead to inflated effect sizes of the intervention, so we will do a calculation of the design effect which involves an estimation of an intracluster correlation (ICC). Estimates of ICC will be imputed using estimates from other included studies that reported ICCs or using external estimates from empirical research. Additionally, we will examine the impact of the clustering using sensitivity analyses.

\section{Dealing with missing data}

Where necessary, we will contact the authors of included studies for which data related to study methods, attrition rates and outcomes are unclear or missing. We will, for example, request number of participants screened, randomly assigned participants, intention to treat, as treated or per protocol samples, dropouts, losses to follow-up or withdrawals. If estimates for the entire study sample are not provided (eg, only estimates for each sex groups are provided), then we will calculate it using available information, including imputing data, where appropriate. We will report all missing outcome data in the data extraction form and risk of bias table, as well as assess the impact of including studies with missing data in sensitivity analysis.

\section{Assessment of heterogeneity}

We will assess heterogeneity and variability among studies in relation to participant, intervention, comparison and outcome information, as well as context and type of screening and its implementation. Where meta-analysis is undertaken, heterogeneity will be assessed by visual inspection of overlap of CIs and statistical methods, that is, $\chi^{2}$ and $\mathrm{I}^{2}$. If the $\chi^{2}$ test has a small $p$ value $(\mathrm{p}<0.1)$ and the $\mathrm{I}^{2}$ statistic is $60 \%$ and above, then this indicates moderate or substantial heterogeneity. ${ }^{18}$ We plan to explore reasons for heterogeneity through subgroup analyses.

\section{Assessing reporting bias}

We will assess the likelihood of reporting bias for each outcome where a sufficient number of studies (more than 10) are included in a meta-analysis. We will use a funnel plot to visually check for asymmetry associated with small-study effects and publication bias. Through sensitivity analysis, we will assess how these factors affect the results and conclusions of the meta-analysis.

\section{Data synthesis}

We will conduct a meta-analysis if the included studies are sufficiently homogenous and if at least two studies of the same design assess the same intervention, comparison and outcome. Outcomes should be at clinically relevant time points after hypertension screening to be analysed; for example, death within 3 months of screening may not be clinically relevant. If the characteristics of included studies are excessively heterogeneous, we will not pool results, but we will present a narrative synthesis of the results, potentially grouping findings by context measures.

We will assess the certainty of the overall evidence for each outcome according to the Grading of Recommendations Assessment, Development and Evaluation (GRADE) approach. ${ }^{19}$ GRADE is for rating the certainty of evidence and grading the strength of recommendations in systematic reviews and it 
includes five criteria for downgrading the certainty of evidence: risk of bias, inconsistency, imprecision, publication bias and indirectness; and three criteria for upgrading the certainty of evidence: large effect, dose response and residual confounding opposing the observed effect. The certainty of evidence will be reported as high, moderate, low or very low. High certainty means that further research is very unlikely to change our confidence in the estimate of effect; moderate certainty means that further research is likely to have an important impact on our confidence in the estimate of effect and may change the estimate; low certainty means that further research is very likely to have an important impact on our confidence in the estimate of effect and is likely to change the estimate; and very low certainty means that we are very uncertain about the estimate. The assessment will be reported in GRADE summary of findings tables. The summary of findings table will include the number of participants and studies included for each outcome, a summary of intervention effect and a measure of the certainty of evidence against GRADE criteria. We will present results for the outcomes listed as 1-7 in the Types of outcome measures section. The seven main outcomes are presented, prioritising clinically important outcomes, followed by adverse effect and health system outcomes. Given the complex nature of the interventions being studied, prespecification of the outcomes is challenging. ${ }^{14}$

\section{Subgroup analysis and investigation of heterogeneity}

Subgroup analyses will be considered according to the following:

- Sex: female or male.

- Age: adolescents (15-24), adults (25-64) and elderly persons $(65+)$.

- Ethnicity: White, Black, Asian or other.

- Setting: rural versus urban; or low-income and middle-income countries versus high-income countries (which will be defined according the World Bank's country classifications by income level) ${ }^{20}$

- Screening tools: electronic, mercury or aneroid.

- Cardiovascular risk factors: overweight or obesity, physical inactivity, dietary factors (eg, sodium or salt intake) and comorbid conditions.

- Study duration: $<6$ months versus $>6$ months or similar.

\section{Sensitivity analysis}

We will conduct sensitivity analysis to explore the influence of various factors, when applicable, on the effect size. We will stratify analyses per publication status and level of risk of bias to determine whether studies with high risk of bias skew the results.

\section{Patient and public involvement}

There was no patient or public involvement in designing the study or writing up the study protocol, but the systematic review is a response to priority setting conducted in collaboration with policy-makers.

Acknowledgements Joy Oliver, Cochrane South Africa, South African Medical Research Council, who assisted with developing the search strategy and scoping the evidence. The authors acknowledge the support of the Cochrane Hypertension Group.

Contributors B-MS drafted the protocol with methods input from SD, IT and JJM and content input from CMB. TK provided both methods and content oversight. All the coauthors have read and approved the final manuscript.

Funding This work is part of the Collaboration for Evidence-Based Healthcare and Public Health (CEBHA+) Research Project (http://cebha-plus.org/Project.html) and was supported by the Federal Ministry for Education and Research (BMBF) (Germany) and Research Networks for Health Innovation in Sub-Saharan Africa.

Competing interests None declared.

Patient consent for publication Not required.

Provenance and peer review Not commissioned; externally peer reviewed.

Open access This is an open access article distributed in accordance with the Creative Commons Attribution Non Commercial (CC BY-NC 4.0) license, which permits others to distribute, remix, adapt, build upon this work non-commercially, and license their derivative works on different terms, provided the original work is properly cited, appropriate credit is given, any changes made indicated, and the use is non-commercial. See: http://creativecommons.org/licenses/by-nc/4.0/.

\section{REFERENCES}

1. Guwatudde D, Nankya-Mutyoba J, Kalyesubula R, et al. The burden of hypertension in sub-Saharan Africa: a four-country cross sectional study. BMC Public Health 2015;15:1-8.

2. Carey RM, Whelton PK; 2017 ACC/AHA Hypertension Guideline Writing Committee. Prevention, detection, evaluation, and management of high blood pressure in adults: synopsis of the 2017 American College of Cardiology/American Heart Association Hypertension Guideline. Ann Intern Med 2018;168:351-8.

3. World Health Organization (WHO). A global brief on hypertension: silent killer, global public health crisis: World Health Day 2013. Geneva: WHO, 2013.

4. Cheung EL, Bell CS, Samuel JP, et al. Race and obesity in adolescent hypertension. Pediatrics 2017;139:e20161433.

5. Kar S, Khandelwal B. Fast foods and physical inactivity are risk factors for obesity and hypertension among adolescent school children in east district of Sikkim, India. Journal of Natural Science. Biology and Medicine 2015;6:356-9.

6. Lloyd-Sherlock P, Ebrahim S, Grosskurth $\mathrm{H}$. Is hypertension the new HIV epidemic? Int J Epidemiol 2014;43:8-10.

7. Ataklte F, Erqou S, Kaptoge S, et al. Burden of undiagnosed hypertension in sub-saharan Africa: a systematic review and metaanalysis. Hypertension 2015;65:291-8.

8. Legorreta AP, Schaff SR, Leibowitz AN, et al. Measuring the effects of screening programs in asymptomatic employees: detection of hypertension through worksite screenings. J Occup Environ Med 2015;57:682-6.

9. Screening Subcommittee, Australian Population Health Development Principal Committee. Population based screening framework: Commonwealth of Australia, 2008.

10. American College of Cardiology Foundation. New ACC/AHA high blood pressure guidelines lower definition of hypertension. 2018.

11. Rohwer A, Pfadenhauer L, Burns J, et al. Series: Clinical Epidemiology in South Africa. Paper 3: logic models help make sense of complexity in systematic reviews and health technology assessments. J Clin Epidemiol 2017;83:37-47.

12. Hunter-Adams J, Yongsi BN, Dzasi K, et al. How to address noncommunicable diseases in urban Africa. Lancet Diabetes Endocrinol 2017;5:932-4.

13. Durao S, Kredo T. Mapping the evidence on population level inteventions to prevent diabetes and hypertension. 2014.

14. Cochrane Effective Practice and Organisation of Care (EPOC): resources for review authors. 2017.

15. Cochrane Effective Practice and Organisation of Care (EPOC), Interrupted time series (ITS) analyses. EPOC Resources for review authors. 2017.

16. Liberati A, Altman DG, Tetzlaff J, et al. The PRISMA statement for reporting systematic reviews and meta-analyses of studies that 
evaluate health care interventions: explanation and elaboration. PLOS Med 2009;6:e1000100.

17. Cochrane Effective Practice and Organisation of Care (EPOC), Suggested risk of bias criteria for EPOC reviews. EPOC Resources fo $r$ review authors. 2017.

18. Higgins J, Altman D, Sterne J. Cochrane handbook for systematic reviews of interventions version 5.1: The Cochrane Collaboration, 2011.
19. Guyatt GH, Oxman AD, Vist GE, et al. GRADE: an emerging consensus on rating quality of evidence and strength of recommendations. BMJ 2008;336:924-6.

20. The World Bank. How does the World Bank classify countries? https://datahelpdesk.worldbank.org/knowledgebase/articles/ 378834-how-does-the-world-bank-classify-countries (Accessed 03 Oct 2018). 